Supporting information to accompany with:

\title{
Promoting Effect of Supports with Oxygen Vacancies as Extrinsic Defects on the Reduction of Iron Oxide
}

\author{
Javier C. Mora ${ }^{1}$, Yoni C.M. Nederstigt ${ }^{2}$, Josephine M. Hill ${ }^{3}$, Sathish Ponnurangam ${ }^{4 *}$
}

1,2,3,4 Department of Chemical \& Petroleum Engineering, Schulich School of Engineering, University of Calgary, 2500 University Dr NW, Calgary, AB, Canada, T2N 1N4

*Corresponding Author

Tel: +1 403210 7342; fax: 1403284 4852; e-mail: sathish.ponnurangam@ucalgary.ca 


\section{S1. Determination of Activation energy}

The activation energy, E, as a function of the reacted fraction was determined by three different methods: maximum rate (Kissinger) ${ }^{1}$, integral ${ }^{2}$, and non-linear Integral (Vyazovkin) ${ }^{3}$. All these methods require TPR patterns, obtained by at least three different heating rates.

1) The equation derived by Kissinger from Eq. (1.12) under the condition of the maximum reaction rate remains as one of the most popular methods in thermal analysis. ${ }^{1}$ This method is based on the variation of peak temperature with heating rate and is given by the following equation:

$$
\ln \left(\frac{\beta}{T_{\max , i}^{2}}\right)=\ln \left(-\frac{A R}{E} f^{\prime}\left(\alpha_{\max }\right)\right)-\frac{E}{R T_{\max , i}}
$$

The left-hand side of equation is plotted against $1 / T_{\max }$ producing a straight line whose slope yield the activation energy $E$. The intercept can be taken as a constant.

2) The Integral methods originates from the approximate solution of the temperature integral. Its form and solution is similar to the Kissinger equation ${ }^{2}$ :

$$
\ln \left(\frac{\beta_{i}}{T_{\alpha, i}^{1.92}}\right)=C-\frac{E}{R T_{\alpha, i}}
$$

4) Finally the non-linear optimization method developed by Vyazovkin ${ }^{3}$ was used to obtain the dependency of $E \alpha$ on the heating rate:

$$
\min \Phi\left(E_{\alpha}\right)=\sum_{i=1}^{n} \sum_{j \neq 1}^{n} \frac{I\left(E_{\alpha} T_{\alpha, i}\right) \beta_{j}}{I\left(E_{\alpha} T_{\alpha, j}\right) \beta_{i}}
$$


where the function I is equal to the temperature (or Arrhenius) integral:

$$
I\left(E_{\alpha} T_{\alpha}\right)=\int_{0}^{T_{\alpha}} \exp \left(\frac{E_{\alpha}}{R T}\right) d T
$$

The temperature integral cannot be solved analytically. Senum and Yang ${ }^{4}$ derived a rational approximation using a ratio of two algebraic polynomials. For all the calculations an eighthdegree rational approximation for this expression was used. This expression allows an accuracy better than $10^{-8 \%} \%$. and can be found in reference. ${ }^{5}$

\section{S2. Modelling TPR profiles}

To model TPR profiles, the following analytical expressions based on the method proposed by Wimmers ${ }^{6}$ were derived

Briefly, for the differential equation (1), separation of variables is possible, leading to:

$$
g(\alpha)=\int_{0}^{\alpha} \frac{d \alpha}{f(\alpha)}=\frac{A}{\beta} \int_{T_{0}}^{T} \exp \left(\frac{-E}{R T}\right) d T=\frac{A}{\beta} I(T)=S(T)
$$

where $I(T)$ is the temperature integral given by equation (S5) and $g(\alpha)$ is the integral form of the reaction model. After deriving this equation, the following expressions are obtained:

$$
\begin{gathered}
\frac{d S(T)}{d T}=\frac{A}{\beta} \exp \left(\frac{-E}{R T}\right) \\
\frac{d^{2} S(T)}{d S^{2}}=\frac{A}{\beta} \exp \left(\frac{-E}{R T}\right)\left[\frac{E}{R T^{2}}\right]=\left[\frac{E}{R T^{2}}\right] \frac{d S(T)}{d T}
\end{gathered}
$$

It can easily be demonstrated that the integral form of the JMAK expression is: 


$$
g(\alpha)=[-\ln (1-\alpha)]^{\frac{1}{n}}=S(T)
$$

So, Equation S9 can be rewritten as:

$$
\alpha=1-\exp \left[S(T)^{n}\right]
$$

Deriving equation S10 and considering that the internal derivative is expressed by equation S6, it follows that:

$$
\frac{d \alpha}{d T}=n \exp \left(-[S(T)]^{n}\right) S(T)^{n-1} \frac{A}{\beta} \exp \left(\frac{-E}{R T}\right)
$$

This final equation describes the reduction rate as a function of temperature under a constant heating rate for one elementary reaction, i.e., a TPR patterns. 


\section{S3. Residual Plots}
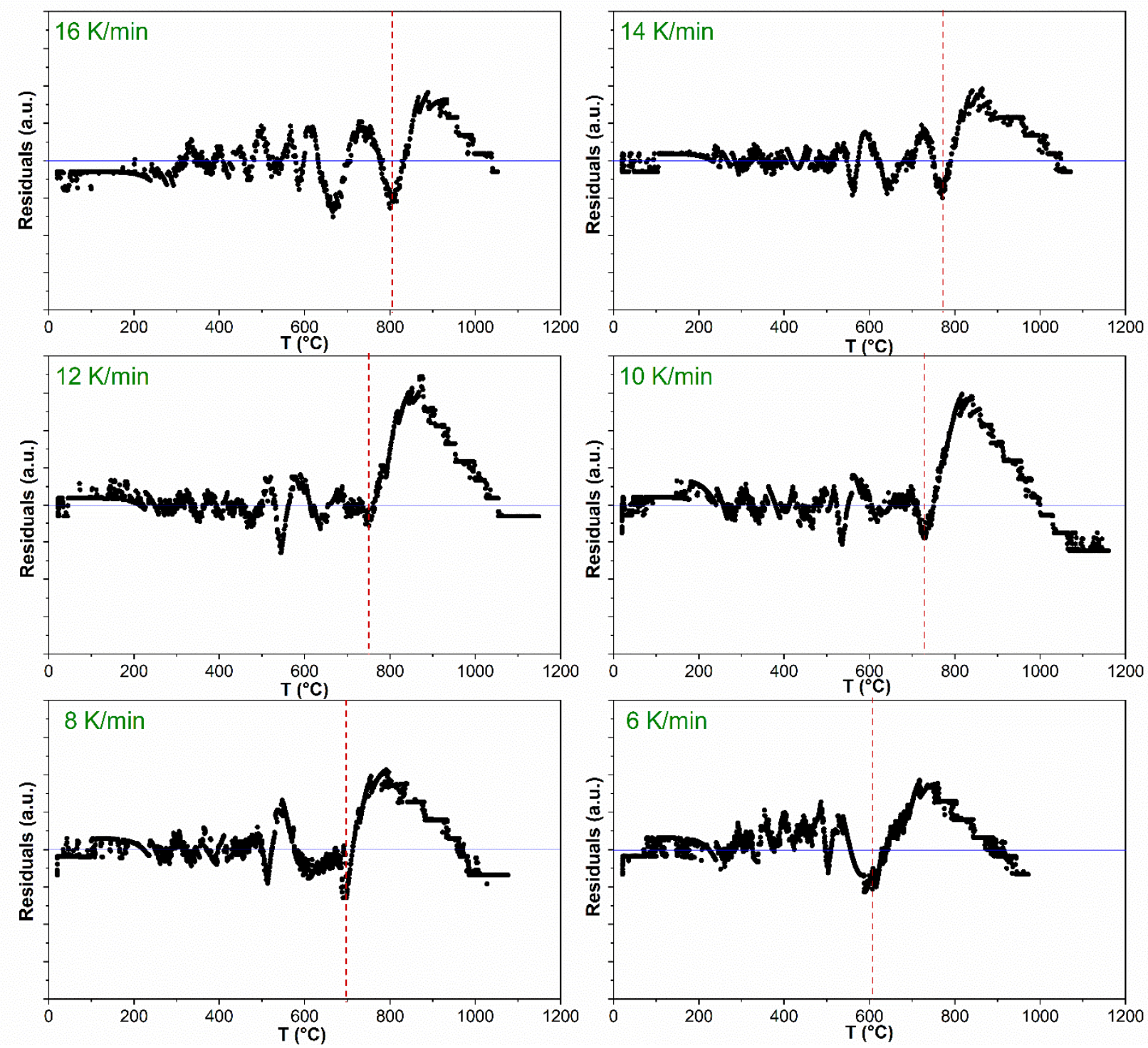

Figure S1. Residual plots for the model-fitting of TPR profiles for the reduction of $\mathrm{Fe}_{2} \mathrm{O}_{3}$ supported on YSZ at different heating rates. The red line indicates the beginning of the high temperature tail. 

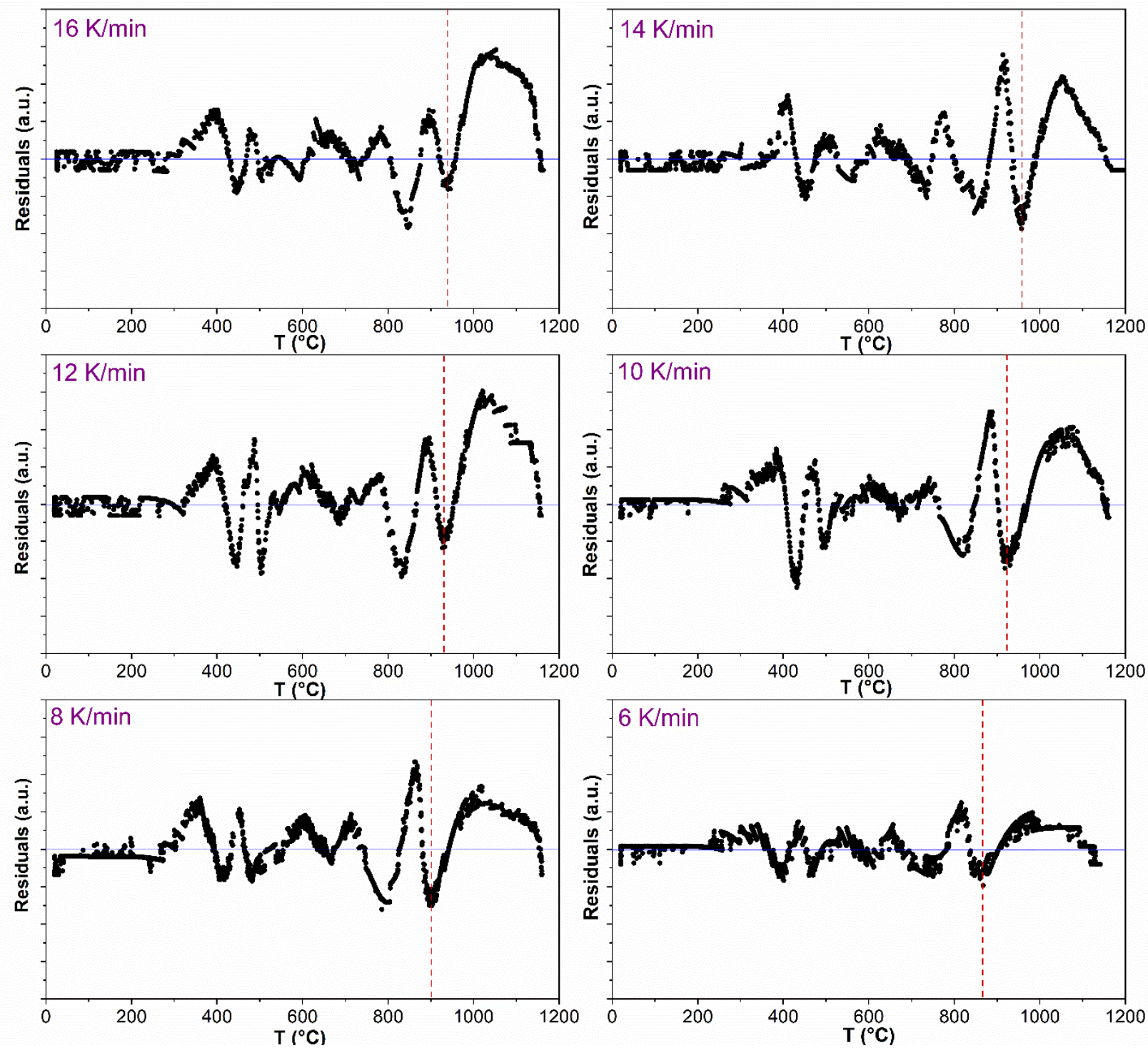

Figure S2. Residual plots for the model-fitting of TPR profiles for the reduction of $\mathrm{Fe}_{2} \mathrm{O}_{3}$ supported on $B C Z Y$ at different heating rates. The red line indicates the beginning of the high temperature tail. 


\section{S4. Discarding other mechanistic models for the reduction of $\mathrm{Fe}_{2} \mathrm{O}_{3} / \mathrm{YSZ}$ and $\mathrm{Fe}_{2} \mathrm{O}_{3} / \mathrm{BCZY}$}

The procedure developed by Wimmers et $\mathrm{al}^{6}$, in which a given peak is fitted with different peak models with the same area (corresponding to equal $\mathrm{H}_{2}$ consumption), was used to rule out the occurrence of other solid-gas reaction mechanisms. We illustrate this procedure in Figure S3, for the model-fitting of one representative deconvoluted peak using different solid-gas reaction mechanisms:
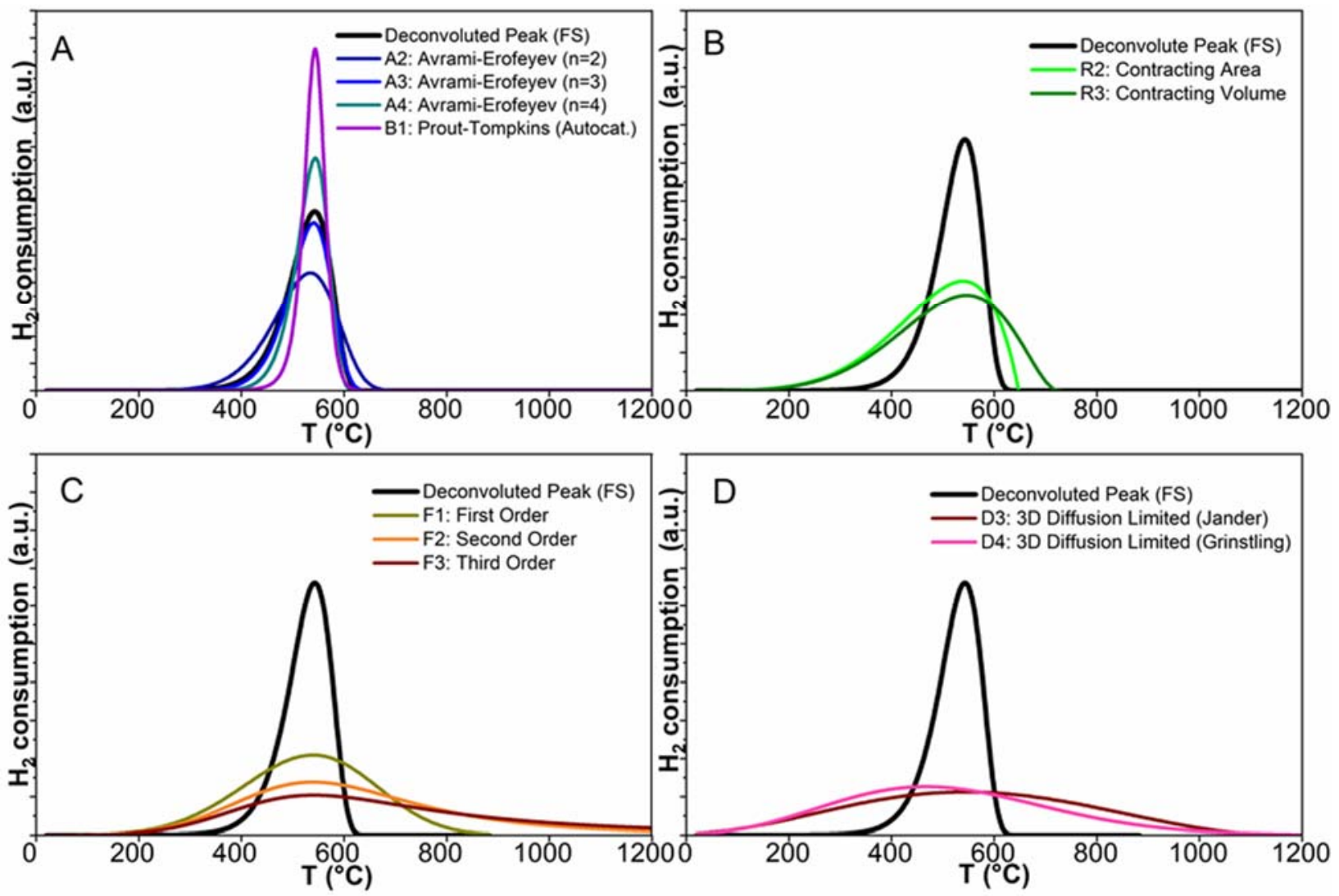

Figure S3. Model-fitting using different solid-gas reaction mechanisms for one representative TPR deconvoluted peak. Representative peak: reduction step $\mathrm{Fe}_{3} \mathrm{O}_{4} \rightarrow \mathrm{FeO}$ for $\mathrm{Fe}_{2} \mathrm{O}_{3} / \mathrm{YSZ}$ at 14 ${ }^{\circ} \mathrm{C} / \mathrm{min}$. Models: nucleation/nuclei-growth and autocatalytic (A), geometrical contraction (B), order reaction $(\mathrm{C})$, and diffusion controlled (D).

As shown in Figure S3, none of the reduction mechanisms apart from nucleation/nuclei growth gives a reasonable fit (The JMAK model indicates that $n=3.05$, which is consistent with Figure S3.A). This procedure was implemented in our calculation routine and repeated for all single 
deconvoluted peaks. The as-obtained results indicate that none of the 48 deconvoluted peaks for $\mathrm{Fe}_{2} \mathrm{O}_{3} / \mathrm{YSZ}$ and $\mathrm{Fe}_{2} \mathrm{O}_{3} / \mathrm{BCZY}$ can be described by any other solid-gas reaction model apart from nucleation/nuclei growth.

\section{S5. Influence of the passivation layer}

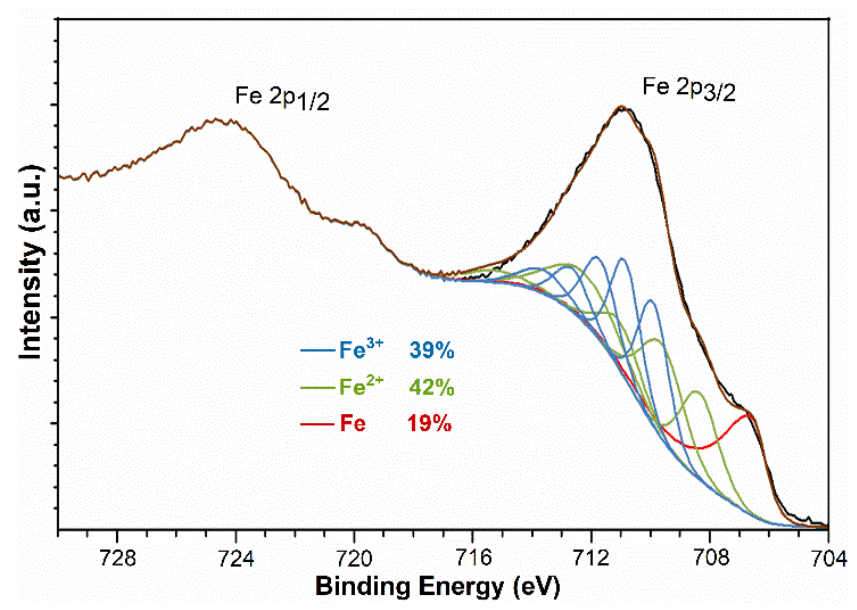

Figure S4: $\mathrm{Fe} 2 \mathrm{p}$ XPS spectrum for fully reduced $\mathrm{Fe}_{2} \mathrm{O}_{3}$ nanoparticles, which were subsequently passivated according to the procedure reported in reference ${ }^{7}$.

The XPS spectra of a reduced iron oxide nanoparticles after the passivation treatment (shown in Figure S4) reveal that a significant fraction of the metallic iron formed was oxidize. The passivation treatment increases the superficial oxidation state of iron from 0 to 2.0 .

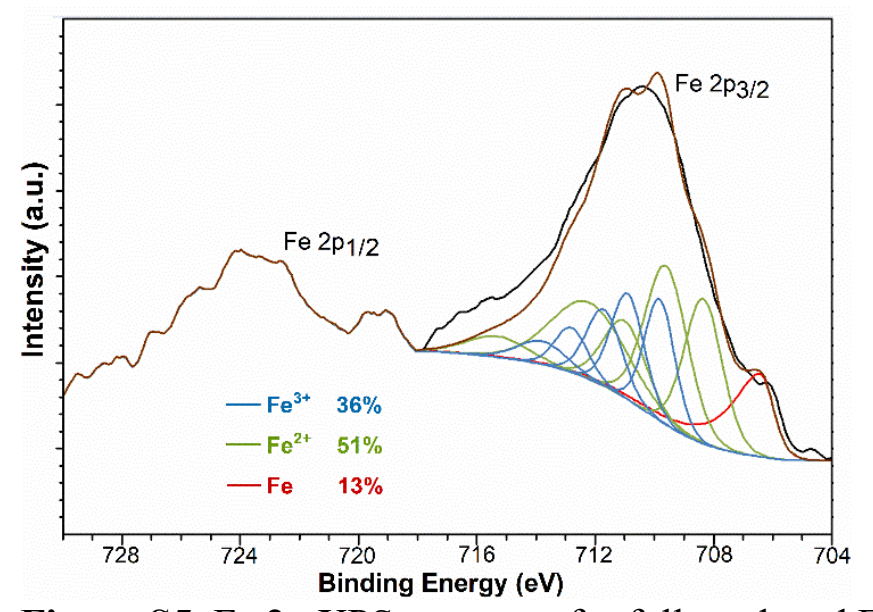

Figure S5. Fe 2p XPS spectrum for fully reduced $\mathrm{Fe}_{2} \mathrm{O}_{3} / \mathrm{YSZ}$, which was subsequently passivated according to the procedure reported in reference ${ }^{7}$. 
XPS spectra of fully reduced $\mathrm{Fe}_{2} \mathrm{O}_{3} / \mathrm{YSZ}$ indicate that the average superficial oxidation state of iron is 2.1. This result is in good agreement with the results obtained for the passivated samples of reduced iron oxide, suggesting that only metallic iron is obtained at the end of the reduction.

\section{References}

(1) Blaine, R. L.; Kissinger, H. E. Homer Kissinger and the Kissinger Equation. Thermochim. Acta 2012, 540, 1-6.

(2) Starink, M. J. The Determination of Activation Energy from Linear Heating Rate Experiments: A Comparison of the Accuracy of Isoconversion Methods. Thermochim. Acta 2003, 404 (1-2), 163-176.

(3) Vyazovkin, S.; Dollimore, D. Linear and Nonlinear Procedures in Isoconversional Computations of the Activation Energy of Nonisothermal Reactions in Solids. J. Chem. Inf. Comput. Sci. 1996, 36 (1), 42-45.

(4) Senum, G. I.; Yang, R. T. Rational Approximations of the Integral of the Arrhenius Function. J. Therm. Anal. 1977, 11 (3), 445-447.

(5) Pérez-Maqueda, L. A.; Criado, J. M. Accuracy of Senum and Yang's Approximations to the Arrhenius Integral. J. Therm. Anal. Calorim. 2000, 60 (3), 909-915.

(6) Wimmers, O. J. Analytical Expressions for Temperature Programmed Reduction Patterns Using Solid-State Kinetics. Thermochim. Acta 1985, 95 (1), 67-72.

(7) Shroff, M. D.; Datye, A. K. The Importance of Passivation in the Study of Iron FischerTropsch Catalysts. Catal. Letters 1996, 37 (1-2), 101-106. 